\title{
Calculated Curves for Groundwave Propagation Over Inhomogeneous Earth With Pronounced Topographical Features
}

\author{
K. Furutsu \\ National Bureau of Standards, Boulder, Colo. \\ (Received December 21, 1964; revised January 28, 1965)
}

\begin{abstract}
Several numerical examples are presented for radio propagation over a three-section path. These are based on formulas which were derived earlier [Furutsu, 1957a, 1957b, 1959, 1963; Furutsu, Wilkerson, and Hartmann, 1964] in which the height and the electrical properties of each section may be different. First the path is assumed to be a land-sea-land path and a sea-land-sea path with no height difference; the attenuation using this model is obtained both for the flat earth and the spherical earth and the values are compared to show the asymptotic approach of these two values at short distances. Second, the effect of the height and width of a mesa is illustrated and the attenuatien values are compared to the values obtained when the obstacle is a knife-edge of the same height. Next the effect of varying the receiver height is presented when the path is either a sea-land-sea path or a land-sea-land path. Finally, examples of the attenuation caused by both a channel and an island having bluffs are given which illustrate the recovery effect as well as the diffraction loss in the vicinity of the coastlines.
\end{abstract}

\section{Introduction}

This paper presents some numerical results obtained assuming a three-section inhomogeneous earth which may be either spherical or flat. It is a continuation of a paper which assumed a twosection earth [Furutsu, Wilkerson, and Hartmann, 1964]. These two papers are based on a theory of radio wave propagation which was developed previously [Furutsu, 1955, 1957a, 1957b, 1959, 1963]. The basic terrain consists of three sections of different heights and electrical properties with a ridge at each section boundary. This terrain is illustrated in figure 1 . Using this model the field may be found over an inhomogeneous earth path consisting of three sections, such as a land-sea-land path having a bluff at each coastline, a two ridge path, or a path including a plateau, etc.

The basic formula of field strength can be given in a form of multiple residue series, which reduces to the ordinary $\mathrm{V}$ an der Pol and Bremmer series in the special case of a homogeneous earth. Also the corresponding flat earth formula has been obtained in an analytical form for the limited case where the earth's surface is smooth and both the transmitter and receiver are on the ground [Furutsu, 1955]. This flat earth formula may be used for short distance paths when the spherical earth formula is poorly convergent.

Figure 1 shows the basic terrain used in this paper, which consists of three sections of different earth radii, $a_{2}, a_{4}$, and $a_{6}$, and different propagation constants, $k_{2}, k_{4}$, and $k_{6}$, respectively, with ridges of radial heights, $a_{3}$ and $a_{5}$ (measured from the earth's center to the tops of the ridges), at two boundaries of the three sections. The transmitter and the receiver are located at the points

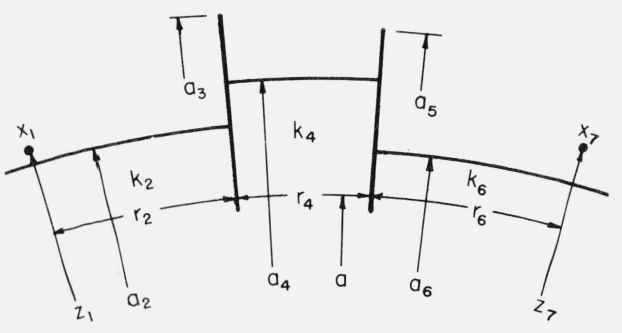

FIGURE 1. The geometry of the basic terrain for equation (1.2). 
$x_{1}$ and $x_{7}$ whose radial heights are $z_{1}$ and $z_{7}$, respectively. Also, the path lengths along these three sections are respectively $r_{2}, r_{4}$, and $r_{6}$, which are assumed to be measured along a mean earth surface of radius $a$.

The attenuation coefficient $A$ is defined in such a way that, if $E$ is the field strength to be obtained,

$$
E=2 A E_{0}, \quad E_{0}=\frac{k_{1}^{2}}{4 \pi\left(r_{2}+r_{4}+r_{6}\right)} e^{-i k_{1}\left(r_{2}+r_{4}+r_{6}\right)}, k_{1}=2 \pi / \lambda,
$$

where $\lambda$ is the wave length in free space. Thus, $E_{0}$ may be regarded as the field strength in free space excited by a dipole of the moment $(4 \pi)^{-1}$ (in Gaussian units).

Then, according to formula (38) in [Furutsu, Wilkerson, and Hartmann, 1964], the attenuation $A$ in this particular case becomes as

$$
\begin{aligned}
A=\Sigma_{t_{2}, t_{4}, t_{6}} \sqrt{\pi\left(c_{2}+c_{4}+c_{6}\right)} e^{-i \pi / 4} f_{t_{2}}\left(y_{12}\right)\left(t_{2}-q_{2}^{2}\right)^{-1} e^{-i c 2 t_{2}} & \left\{q_{2} f_{t_{2}}^{\prime}\left(y_{32}\right) f_{t_{4}}\left(y_{34}\right)-q_{4} f_{t_{4}}^{\prime}\left(y_{34}\right) f_{t_{2}}\left(y_{32}\right)\right\} \\
\times\left(y_{2}-y_{4}+t_{2}-t_{4}\right)^{-1}\left(t_{4}-q_{4}^{2}\right)^{-1} e^{-i c 4 t_{4}} & \left\{q_{4} f_{t_{4}}^{\prime}\left(y_{54}\right) f_{t_{6}}\left(y_{56}\right)-q_{6} f_{t_{6}}^{\prime}\left(y_{56}\right) f_{t_{4}}\left(y_{54}\right)\right\} \\
& \times\left(y_{4}-y_{6}+t_{4}-t_{6}\right)^{-1}\left(t_{6}-q_{6}^{2}\right)^{-1} e^{-i c 6 t_{6}} f_{t_{6}}\left(y_{76}\right),
\end{aligned}
$$

where $c_{2}, c_{4}$, and $c_{6}$ are the numerical distances corresponding to the distances $r_{2}, r_{4}$, and $r_{6}$, i.e.,

$$
c_{2}=\left(r_{2} / a\right)\left(k_{1} a / 2\right)^{1 / 3}, \quad c_{4}=\left(r_{4} / a\right)\left(k_{1} a / 2\right)^{1 / 3}, \quad c_{6}=\left(r_{6} / a\right)\left(k_{1} a / 2\right)^{1 / 3},
$$

and

$$
y_{i j}=y_{i}-y_{j}
$$

where $y_{i}(i=1,2, \ldots, 7)$ are the numerical heights defined by

$$
\begin{gathered}
y_{1}=k_{1}\left(z_{1}-a\right)\left(2 / k_{1} a\right)^{1 / 3}, \quad y_{7}=k_{1}\left(z_{7}-a\right)\left(2 / k_{1} a\right)^{1 / 3}, \\
y_{i}=k_{1}\left(a_{i}-a\right)\left(2 / k_{1} a\right)^{1 / 3}, \quad i=2,3, \ldots ., 6 .
\end{gathered}
$$

Here the set of $t_{m}(m=2,4,6)$ stands for the roots of the equation

$$
W^{\prime}\left(t_{m}\right)-q_{m} W\left(t_{m}\right)=0
$$

where $W^{\prime}(t)$ is the first derivative of the function $W(t)$ defined by

$$
W(-t)=(\pi t / 3)^{1 / 2} e^{-i 2 \pi / 3} H_{1 / 3}^{(2)}\left(\frac{2}{3} t^{3 / 2}\right)
$$

and

$$
q_{m}=-i\left(k_{1} a / 2\right)^{1 / 3}\left(k_{1} / k_{m}^{\prime}\right)
$$

with

$$
k_{m}^{\prime}=\left\{\begin{array}{lc}
k_{m}^{2} / \sqrt{k_{m}^{2}-k_{1}^{2}}, & \text { Vertical Polarization } \\
k_{1}^{2} / \sqrt{k_{m}^{2}-k_{1}^{2}}, & \text { Horizontal Polarization }
\end{array}\right.
$$


Also

$$
\begin{gathered}
f_{t_{m}}(y)=W\left(t_{m}-y\right) / W\left(t_{m}\right), \\
f_{t_{m}}^{\prime}(y)=W^{\prime}\left(t_{m}-y\right) / W^{\prime}\left(t_{m}\right)=q_{m}^{-1} W^{\prime}\left(t_{m}-y\right) / W\left(t_{m}\right),
\end{gathered}
$$

where $f_{t_{m}}(y)$ is the ordinary height gain function. From these definitions it follows that

$$
f_{t_{m}}(0)=f_{t_{m}}^{\prime}(0)=1
$$

The corresponding attenuation coefficient in the flat earth approximation can be given in terms of the following functions:

$$
\begin{gathered}
\mathscr{E}(z)=\frac{2}{\sqrt{\pi}} e^{z^{2}} \int_{z}^{\infty} e^{-t^{2}} d t, \quad \mathscr{E}(z, \alpha)=\left(\frac{2}{\sqrt{\pi}}\right)^{2} e^{\left(1+\alpha^{2}\right) z^{2}} \int_{z}^{\infty} d t_{1} e^{-t_{1}^{2}} \int_{\alpha t_{1}}^{\infty} e^{-t_{2}^{2}} d t_{2}, \\
\mathscr{E}(z, \beta, \alpha)=\left(\frac{2}{\sqrt{\pi}}\right)^{3} e^{\left\{1+\beta^{2}\left(1+\alpha^{2}\right)\right\} z^{2}} \int_{z}^{\infty} d t_{1} e^{-t_{1}^{2}} \int_{\beta t_{1}}^{\infty} d t_{2} e^{-t_{2}^{2}} \int_{\alpha t_{2}}^{\infty} e^{-t_{3}^{2}} d t_{3}, \\
\chi(z, \beta, \alpha)=\left(\frac{2}{\sqrt{\pi}}\right)^{3} e^{\left(1+\alpha^{2}+\beta^{2}\right) z^{2}} \int_{z}^{\infty} d t_{1} e^{-t_{1}^{2}} \int_{\beta t_{1}}^{\infty} e^{-t_{2}^{2}} d t_{2} \int_{\alpha t_{1}}^{\infty} e^{-t_{3}^{2}} d t_{3},
\end{gathered}
$$

$\mathscr{E}^{(n)}(z)=(\partial / \partial z)^{n} \mathscr{E}(z)$

$$
\begin{gathered}
\mathscr{E}^{(n)}(z, \alpha)=(\partial / \partial z)^{n} \mathscr{E}(z, \alpha)=2\left(1+\alpha^{2}\right)\left\{z \mathscr{E}^{(n-1)}(z, \alpha)+(n-1) \mathscr{E}^{(n-2)}(z, \alpha)\right\}-(2 / \sqrt{\pi}) \alpha^{n-1} \mathscr{E}^{(n-1)}(\alpha z), \\
\chi^{(1)}(z, \beta, \alpha)=(\partial / \partial z) \chi(z, \beta, \alpha)=2\left(1+\alpha^{2}+\beta^{2}\right) z \chi(z, \beta, \alpha)-(2 / \sqrt{\pi}) \mathscr{E}(\alpha z) \mathscr{E}(\beta z) .
\end{gathered}
$$

with the relations

$$
\begin{aligned}
& \mathscr{E}(z, \alpha)=\mathscr{E}(z) \mathscr{E}(\alpha z)-\mathscr{E}\left(\alpha z, \alpha^{-1}\right), \quad \alpha>0 \\
& \qquad \begin{aligned}
\mathscr{E}(z) \mathscr{E}(\beta z, \alpha)-\mathscr{E}(z, \beta, \alpha)=\mathscr{E}(\alpha \beta z) \mathscr{E}\left(\beta z, \beta^{-1}\right)-\mathscr{E}\left(\alpha \beta z, \alpha^{-1}, \beta^{-1}\right) \\
=\chi\left(\beta z, \beta^{-1}, \alpha\right), \quad \alpha, \beta>0 .
\end{aligned}
\end{aligned}
$$

The attenuation coefficient, $A$, is given in appendix $\mathrm{A}$ for the general case. In the special case of $k_{2}^{\prime}=k_{6}^{\prime}$, which may be practically most important (land-sea-land and sea-land-sea paths), the attenuation is given by

$$
\begin{aligned}
& A=-\frac{\sqrt{\pi}}{2} \int \mathscr{E}(1)\left(i \sqrt{\rho_{2}}\right)+2 i \sqrt{\rho_{4}}\left(\frac{k_{2}^{\prime}}{k_{4}^{\prime}+k_{2}^{\prime}}\right)^{2}\left[\frac{2}{\pi} \tan ^{-1} \sqrt{\frac{m_{4}}{m_{2} m_{6}}} \mathscr{E}\left(i \sqrt{\rho_{4}}\right)\right. \\
& -\mathscr{E}\left(i \sqrt{m_{2} \rho_{4}}, \sqrt{\left(m_{4}+m_{6}\right) / m_{2}}\right)-\mathscr{E}\left(i \sqrt{m_{6} \rho_{4}}, \sqrt{\left(m_{2}+m_{4}\right) / m_{6}}\right) \\
& +\mathscr{E}\left(i \sqrt{m_{2} \rho_{4}}, \sqrt{m_{6} / m_{2}}, \sqrt{m_{4} / m_{6}}\right)+\mathscr{E}\left(i \sqrt{m_{6} \rho_{4}}, \sqrt{m_{2} / m_{6}}, \sqrt{m_{4} / m_{2}}\right) \\
& +\left(k_{4}^{\prime} / k_{2}^{\prime}\right)\left\{\left(\mathscr{E}\left(i \sqrt{\rho_{4}\left(m_{4}+m_{6}\right)}\right)-\mathscr{E}\left(i \sqrt{m_{6} \rho_{4}}, \sqrt{m_{4} / m_{6}}\right)\right) \mathscr{E}\left(i \sqrt{m_{2} \rho_{2}}\right)\right. \\
& \left.+\left(\mathscr{E}\left(i \sqrt{\rho_{4}\left(m_{4}+m_{2}\right)}\right)-\mathscr{E}\left(i \sqrt{m_{2} \rho_{4}}, \sqrt{m_{4} / m_{2}}\right)\right) \mathscr{E}\left(i \sqrt{m_{6} \rho_{2}}\right)\right\}
\end{aligned}
$$




$$
\begin{aligned}
& \left.+\left(k_{4}^{\prime} / k_{2}^{\prime}\right)^{2} \mathscr{E}\left(i \sqrt{m_{2} \rho_{2}}\right) \mathscr{E}\left(i \sqrt{m_{4} \rho_{4}}\right) \mathscr{E}\left(i \sqrt{m_{6} \rho_{2}}\right)\right] \\
& +i \sqrt{\rho_{2}}\left[\frac { 1 } { 2 } ( \frac { k _ { 4 } ^ { \prime } - k _ { 2 } ^ { \prime } } { k _ { 4 } ^ { \prime } + k _ { 2 } ^ { \prime } } ) \left\{\frac{2}{\pi} \tan ^{-1} \sqrt{\frac{m_{4}}{m_{2} m_{6}}} \mathscr{E}^{(2)}\left(i \sqrt{\rho_{2}}\right)\right.\right. \\
& +\left(\mathscr{E}\left(i \sqrt{\rho_{2}\left(m_{4}+m_{6}\right)}\right)-\mathscr{E}\left(i \sqrt{m_{4} \rho_{2}}, \sqrt{m_{6} / m_{4}}\right)\right) \mathscr{E}(2)\left(i \sqrt{m_{2} \rho_{2}}\right) \\
& +\left(\mathscr{E}\left(i \sqrt{\rho_{2}\left(m_{4}+m_{2}\right)}\right)-\mathscr{E}\left(i \sqrt{m_{4} \rho_{2}}, \sqrt{m_{2} / m_{4}}\right)\right) \mathscr{E}^{(2)}\left(i \sqrt{m_{6} \rho_{2}}\right) \\
& +2\left(\chi\left(i \sqrt{m_{4} \rho_{2}}, \sqrt{m_{6} / m_{4}}, \sqrt{m_{2} / m_{4}}\right)+i \sqrt{m_{4} \rho_{2}} \chi^{(1)}\left(i \sqrt{m_{4} \rho_{2}}, \sqrt{m_{6} / m_{4}}, \sqrt{m_{2} / m_{4}}\right)\right) \\
& -2 i\left(\sqrt{\left(m_{4}+m_{6}\right) \rho_{2}} \mathscr{E}^{(1)}\left(i \sqrt{\rho_{2}\left(m_{4}+m_{6}\right)}, \sqrt{m_{2} /\left(m_{4}+m_{6}\right)}\right)\right. \\
& \left.+\sqrt{\left(m_{4}+m_{2}\right) \rho_{2}} \mathscr{E}^{(1)}\left(i \sqrt{\rho_{2}\left(m_{4}+m_{2}\right)}, \sqrt{\left.m_{6} /\left(m_{4}+m_{2}\right)\right)}\right)\right\} \\
& +\left\{2\left(\frac{k_{2}^{\prime}}{k_{4}^{\prime}+k_{2}^{\prime}}\right)^{2}+\frac{k_{2}^{\prime}-k_{4}^{\prime}}{k_{2}^{\prime}+k_{4}^{\prime}}-1\right\}\left\{\mathscr{\mathscr { C }}\left(i \sqrt{\rho_{2}\left(m_{4}+m_{6}\right)}, \sqrt{m_{2} /\left(m_{4}+m_{6}\right)}\right)\right. \\
& +\mathscr{E}\left(i \sqrt{\rho_{2}\left(m_{4}+m_{2}\right)}, \sqrt{\left.m_{6} /\left(m_{4}+m_{2}\right)\right)}\right\} \\
& \left.\left.-2\left(\frac{k_{2}^{\prime}}{k_{4}^{\prime}+k_{2}^{\prime}}\right)^{2}\left\{\frac{2}{\pi} \tan ^{-1} \sqrt{\frac{m_{4}}{m_{2} m_{6}}} \mathscr{E}\left(i \sqrt{\rho_{2}}\right)+\chi\left(i \sqrt{m_{4} \rho_{2}}, \sqrt{m_{6} / m_{4}}, \sqrt{m_{2} / m_{4}}\right)\right\}\right]\right\} .
\end{aligned}
$$

Here

$$
\begin{aligned}
& m_{2}=r_{2} / r, \quad m_{4}=r_{4} / r, \quad m_{6}=r_{6} / r, \quad r=r_{2}+r_{4}+r_{6}, \\
& \rho_{2}=-i\left(k_{1} / k_{2}^{\prime}\right)^{2} k_{1} r / 2, \quad \rho_{4}=-i\left(k_{1} / k_{4}^{\prime}\right)^{2} k_{1} r / 2,
\end{aligned}
$$

and $\rho_{2}$ and $\rho_{4}$ are the total Sommerfeld's numerical distances measured by the ground constants $k_{2}$ and $k_{4}$, respectively.

Using the identities

$$
\begin{aligned}
& \mathscr{E}\left(i \sqrt{m_{2} \rho_{4}}, \sqrt{m_{6} / m_{2}}, \sqrt{m_{4} / m_{6}}\right)-\mathscr{E}\left(i \sqrt{m_{2} \rho_{4}}\right) \mathscr{E}\left(i \sqrt{m_{6} \rho_{4}}, \sqrt{m_{4} / m_{6}}\right) \\
+ & \mathscr{E}\left(i \sqrt{m_{6} \rho_{4}}, \sqrt{m_{2} / m_{6}}, \sqrt{m_{4} / m_{2}}\right)-\mathscr{E}\left(i \sqrt{m_{6} \rho_{4}}\right) \mathscr{E}\left(i \sqrt{m_{2} \rho_{4}}, \sqrt{m_{4} / m_{2}}\right) \\
= & -\chi\left(i \sqrt{m_{6} \rho_{4}}, \sqrt{m_{2} / m_{6}}, \sqrt{m_{4} / m_{6}}\right)-\chi\left(i \sqrt{m_{2} \rho_{4}}, \sqrt{m_{6} / m_{2}}, \sqrt{m_{4} / m_{2}}\right) \\
= & \chi\left(i \sqrt{m_{4} \rho_{4}}, \sqrt{m_{6} / m_{4}}, \sqrt{m_{2} / m_{4}}\right)-\mathscr{E}\left(i \sqrt{m_{2} \rho_{4}}\right) \mathscr{E}\left(i \sqrt{m_{4} \rho_{4}}\right) \mathscr{E}\left(i \sqrt{m_{6} \rho_{4}}\right),
\end{aligned}
$$

the formula (1.12) in the special case of $k_{2}^{\prime}\left(=k_{6}^{\prime}\right)=k_{4}^{\prime}$ can be shown to reduce to that for a homogeneous earth of the ground constant $k_{2}$. The analytic expansions of the functions used are treated in appendix B.

The Sommerfeld numerical distances $\rho_{2}$ and $\rho_{4}$ introduced in (1.13) and the numerical distances $c_{2}, c_{4}$, and $c_{6}$ defined by (1.3) are related by

$$
\rho_{2}=i q_{2}^{2}\left(c_{2}+c_{4}+c_{6}\right), \quad \rho_{4}=i q_{4}^{2}\left(c_{2}+c_{4}+c_{6}\right)
$$


When the frequency is low enough so that $\epsilon_{m}$ is much less than $18 \sigma_{m} / f$, the Sommerfeld numerical distance is given by

$$
\rho_{m}=(5 \pi / 27)\left(f^{2} / \sigma_{m}\right)\left(r_{2}+r_{4}+r_{6}\right) \text {. }
$$

In the above, $f$ is the frequency in $\mathrm{Mc} / \mathrm{s}, \sigma_{m}$ is the conductivity in millimho/m and $r_{i}$ is the distance in $\mathrm{km}$.

In concluding this section, it may be noticed that the theory is valid independent of the incident angles of wave on the boundary lines of sections, except in the immediate vicinities of the boundaries. On the other hand, it does not take into account the possible wave components which are reflected several times between the two boundaries of sections (fig. 1) and are finally propagated to the receiver. In the case of mixed paths (smooth earth), the attenuation also has been obtained in terms of convolution integrals [Feinberg, 1944, 1946, 1959; Bremmer, 1954; Wait and Householder, 1956, 1957; Wait, 1961], and is usually computed by a numerical method.

\section{Three-Section Mixed Paths}

In figure $2 \mathrm{a}$, the flat earth value (broken line) of the attenuation is compared to the spherical earth value (solid line) for a sea-land-sea path (curve 1) and also a land-sea-land path (curve 4).
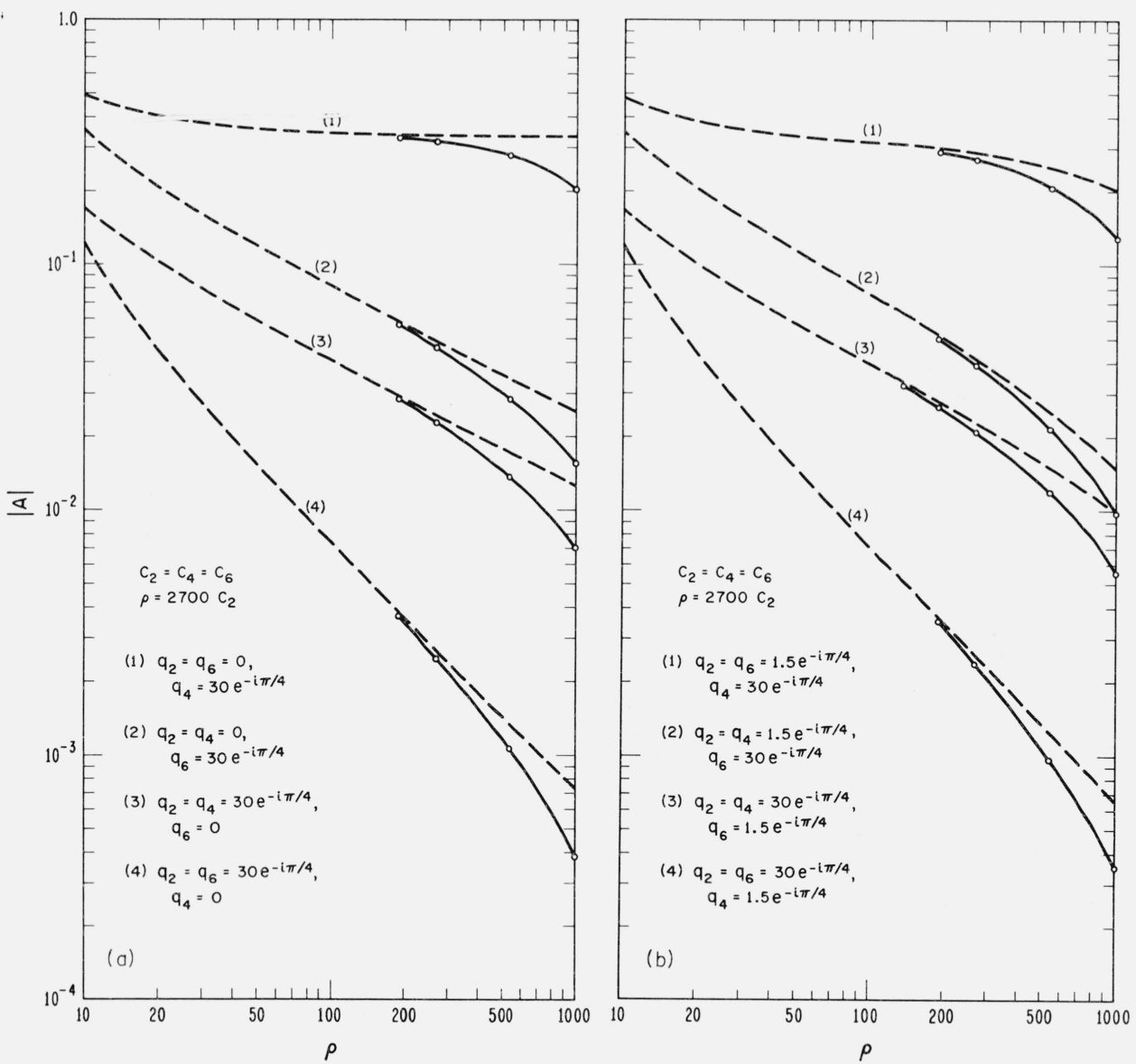

Figures 2a and $2 \mathrm{~b}$. Comparison of the flat earth values of the attenuation to the corresponding spherical earth values for various combinations of the ground constants, when $r_{2}=r_{4}=r_{6}$ and the sea has the constant (a) $\mathrm{q}=0$, (b) $\mathrm{q}=1.5 e^{-i \pi / 4}$. 
The graph is plotted against the total Sommerfeld numerical distance, $\rho$, measured using the conductivity of the land, $\sigma_{L},\left(\epsilon_{L} \ll \sigma_{L} / f\right)$ when $r_{2}=r_{4}=r_{6}$. The sea is assumed to be perfectly conducting and the ground constant of the land is assumed to be $q=30 e^{-i \pi / 4}$. In this figure, the curves 2 and 3 are also shown when the paths are reduced to the mixed paths of two sections caused by putting $q_{2}=q_{4}$.

In figure $2 \mathrm{~b}$, the attenuations are found for the same paths on the same conditions as in figure 2a except that instead of a perfectly conducting sea the ratio of the sea conductivity, $\sigma_{s}$, to that of the land, $\sigma_{L}$, is assumed to be 400 (this gives the ground constant $q=1.5 e^{-i \pi / 4}$ for the sea parts). As in figure $2 \mathrm{a}$, the curves are also shown for the associated two-section paths.

As expected the spherical earth value approaches the flat earth value asymptotically at short distances in both figures.

Figures $3 \mathrm{a}$ and $3 \mathrm{~b}$ illustrate a set of curves of the attenuation and phase for land-sea-land paths when the earth is flat and $\sigma_{s} / \sigma_{L}=400$. They are displayed versus the total Sommerfeld numerical distance measured by using the land conductivity $\sigma_{L}$, for various values of the parameter $N=\left(r_{2}+r_{6}\right)$ $/ r_{4}$ in the particular case of $r_{2}=r_{6}$.

It may be noticed from figure $3 \mathrm{~b}$ that, in the case of the flat earth approximation, the phase delay of the attenuation coefficient for a land-sea-land path can exceed $\pi$ for $\rho \gg 1$ and $N \ll 1$, when the finite conductivity of the sea is taken into account and the corresponding numerical distance of the sea part becomes of the order of magnitude of 1 or greater. A similar situation can also be seen for a land-sea path, a mixed path of two sections, on the same conditions. For comparison, the attenuation and the phase in the latter case of land-sea paths are also displayed in figures $4 \mathrm{a}$ and $4 \mathrm{~b}$ on the same condition, $\sigma_{s} / \sigma_{L}=400$, as in figures $3 \mathrm{a}$ and $3 \mathrm{~b}$, where $N=r_{2} / r_{4}$, the ratio of the land part to the sea part, and $\rho$ is the total Sommerfeld numerical distance measured by using the land conductivity (refer to the equations with $r_{6}=0$ in fig. $3 \mathrm{a}$ ).
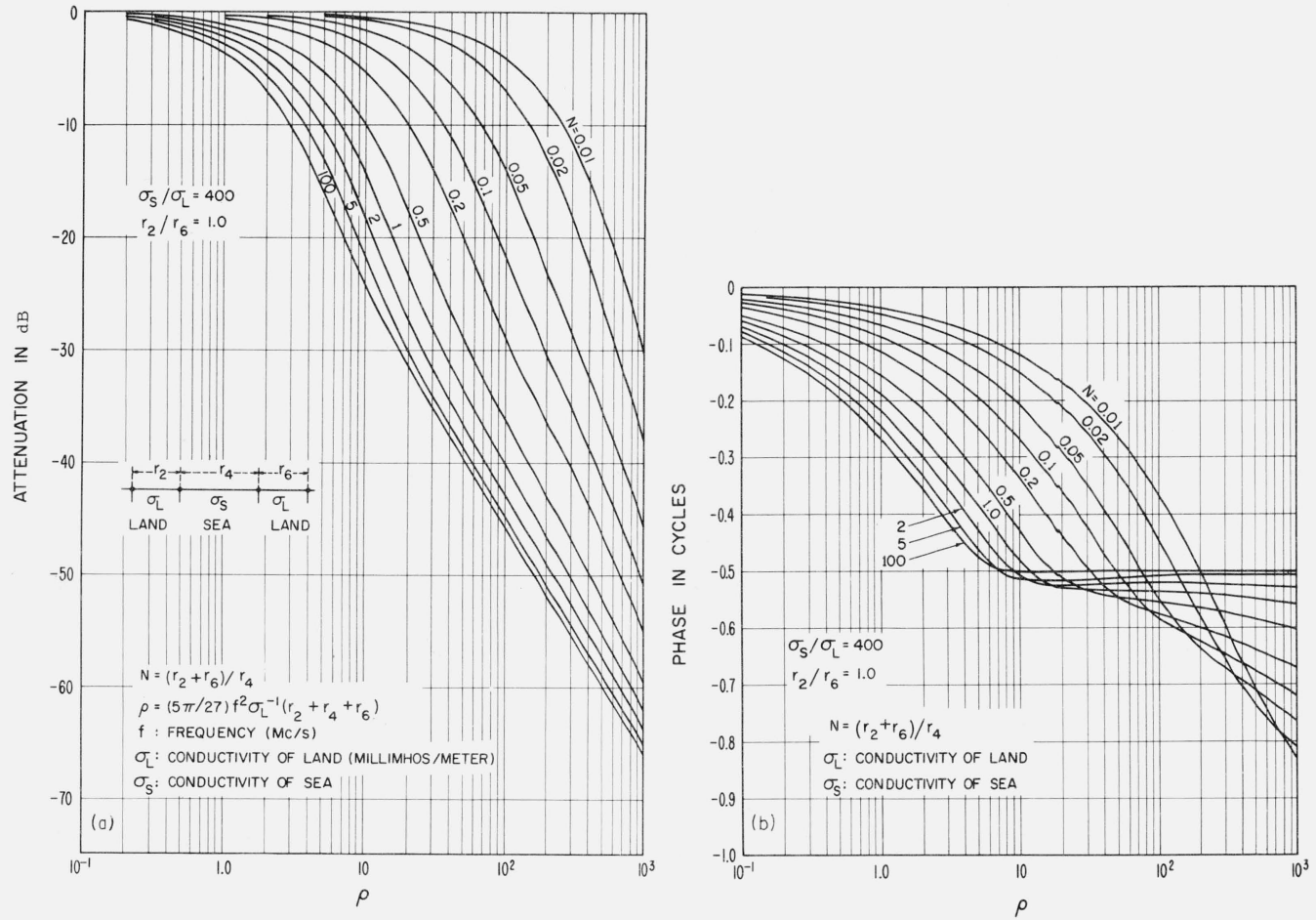

FigurEs 3a and 3b. Flat earth values of $(a)$ the attenuation and $(b)$ the phase delay for land-sea-land paths versus the total Sommerfeld's numerical distance $\rho$ (measured by the land conductivity $\sigma_{L}$ ) for various values of $\mathrm{N}=\left(\mathrm{r}_{2}+\mathrm{r}_{6}\right) / \mathrm{r}_{4}$, when $\sigma_{\mathrm{s}} / \sigma_{\mathrm{L}}=400$ and $\mathrm{r}_{2} / \mathrm{r}_{6}=1$. 

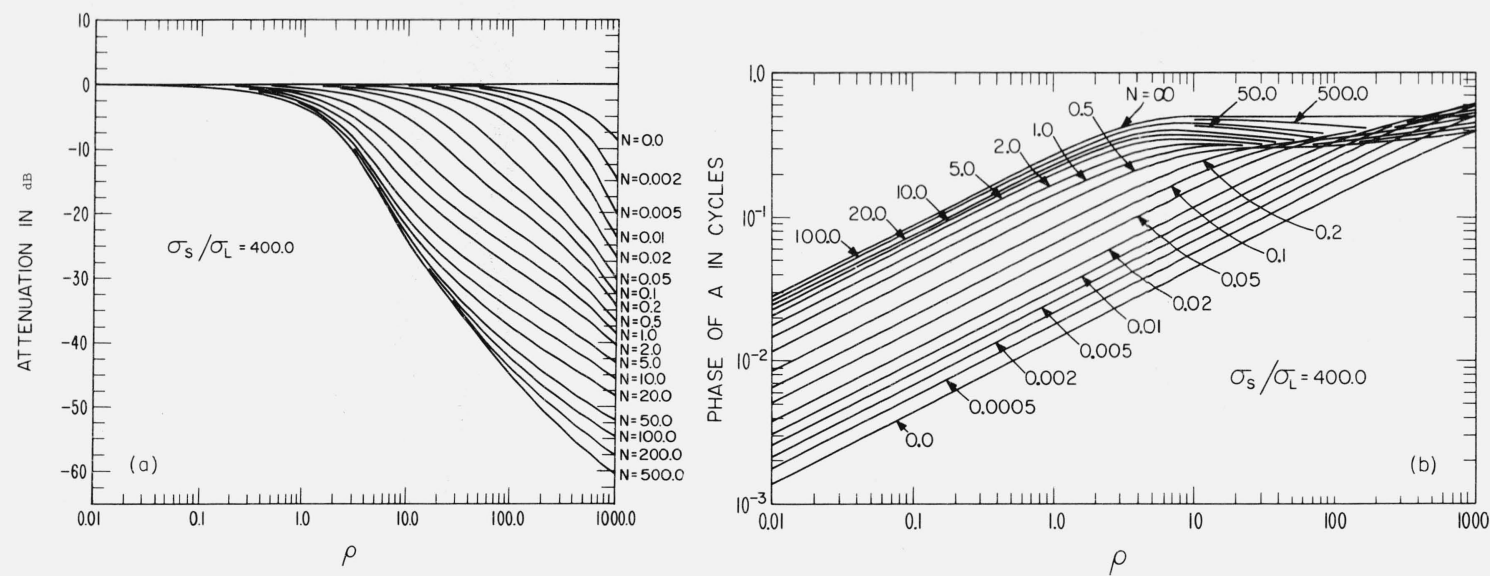

Figures 4a and 4b. Flat earth values of (a) the attenuation and (b) the phase delay for land-sea paths, versus the total Sommerfeld's numerical distance $\rho$ (measured by the land conductivity $\sigma_{\mathrm{L}}$ ) for various values of $\mathrm{N}$, the ratio of the land part to the sea part, when $\sigma_{\mathrm{s}} / \sigma_{\mathrm{L}}=400$.

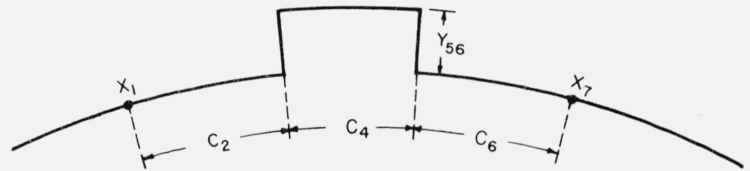

FigURE 5. The geometry of the mesa for figures $6 a$ and $6 b$.
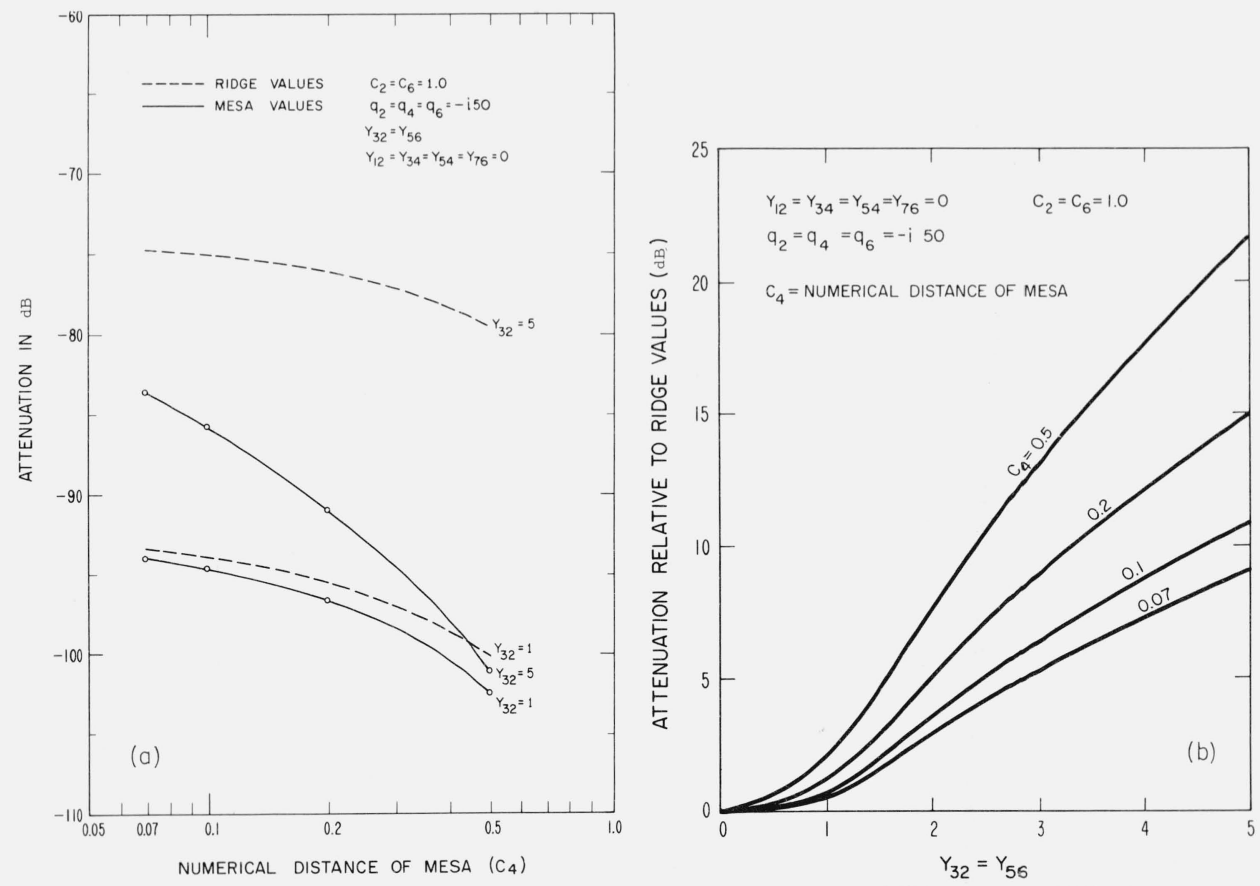

NUMERICAL HEIGHT OF MESA

FIGURE 6a. The attenuation caused by a mesa versus the mesa length compared to the attenuation caused by a ridge of the same height.

Figure 6b. The attenuation caused by a mesa relative to the corresponding ridge value versus its height for various values of the mesa length (the absolute values are displayed). 


\section{Effect of a Mesa}

In order to illustrate the effect of a mesa the terrain is considered to be as shown in figure 5 . The distances on each side of the mesa, $c_{2}$ and $c_{6}$, are both equal to 1 and the transmitter and the receiver are both on the ground. In figure 6 a the attenuation is illustrated versus the numerical width of the mesa, $c_{4}$, for its numerical height, $y_{32}=y_{56}=1$ and 5 .

Also shown in this figure is the value of the attenuation which would be obtained if a ridge (broken line) of the same height as the mesa (solid line) were placed at the midpoint of the path with the same total length. It definitely shows the obstacle gain (the higher the obstacle the smaller the attenuation), and also that the effect of the mesa width becomes greater with increasing height.

The latter situation is also illustrated in figure $6 \mathrm{~b}$, where the attenuation relative to the corresponding ridge value is shown versus the numerical height of the meas for various values of its width with the same values of other parameters as in figure $6 \mathrm{a} .^{1}$

\section{Effect of Varying Receiver Height}

In the previous paper [Furutsu, Wilkerson, and Hartmann, 1964] the variation of the field strength was illustrated when the receiver was raised and the path was a mixed path of two sections (sea-land). A similar situation is illustrated in figure 7 for a mixed path consisting of three sections.

In figure $8 \mathrm{a}$, a path is selected corresponding to a land-sea-land path having the numerical distances $c_{2}=10, c_{4}=1.0$, and $c_{6}=0.7$ with the ground constants $q_{2}=q_{6}=-i 50$ and $q_{4}=-i 10^{-6}$ and, keeping the transmitter $\left(x_{1}\right)$ on the ground, the numerical height $y_{76}$ of the receiver $\left(x_{7}\right)$ is changed; the attenuation is shown by a solid line, while the broken and the chain lines are the associated mixed path values of two sections and the homogeneous earth values for $q_{2}=-i 50$, $q_{4}=q_{6}=-i 10^{-6}$, and $q_{2}=q_{4}=q_{6}=-i 50$, respectively. The two optical boundary points of this path are found to be at $y_{76}=(0.7)^{2}$ and $(1.7)^{2}$. However, as is seen, the three section values approach the two section values and then the homogeneous earth values at much higher points than these optical boundary points. It also shows some oscillation, which may be interpreted as an interference of the principal wave with the waves induced at the section boundaries.

Figure $8 \mathrm{~b}$ shows a similar illustration for a sea-land-sea path with the same values of the parameters involved except that $q_{2}=q_{6}=-i 10^{-6}$ and $q_{4}=-i 50$.

\section{Examples of Field Strength When the Obstacle Is an Island or a Channel}

In figure 9, a sea-land-sea path (island) is considered where the height of the island is $500 \mathrm{~m}$. The transmitter and the receiver are both assumed to be on the ground, the wave frequency is 1 $\mathrm{Mc} / \mathrm{s}$, and vertical polarization is assumed. The island is $200 \mathrm{~km}$ from the transmitter, and the electrical constants of the land are $\epsilon_{L}=4, \sigma_{L}=10 \mathrm{millimho} / \mathrm{m}$ and those of the sea are $\epsilon_{s}=80$, $\sigma_{s}=4000$ millimho/m.

${ }^{1}$ When the frequency is $300 \mathrm{Mc} / \mathrm{s}$, the value $c_{4}=0.5$ corresponds to the distance $14.2 \mathrm{~km}$ and the value $Y_{32}=5$ to the height $238 \mathrm{~m}$.

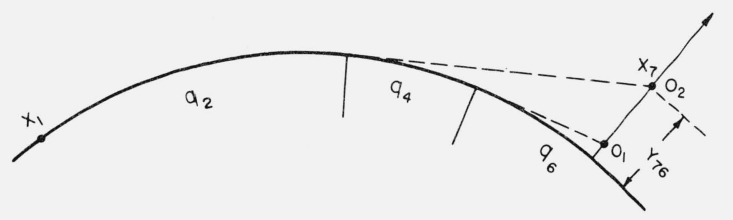

FIGURE 7. The geometry and the optical boundary points for figures $8 a$ and $8 b$. 

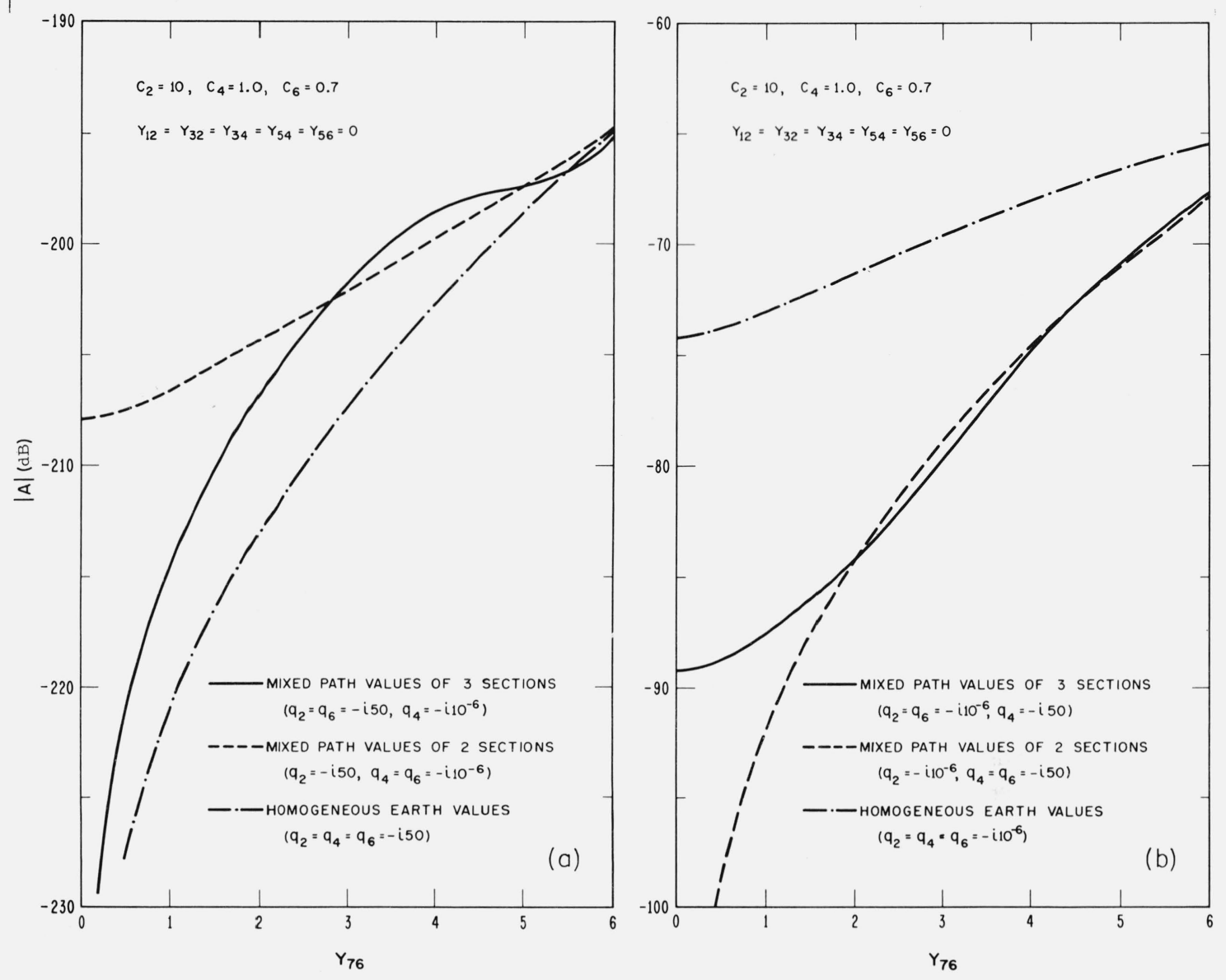

FIGURES $8 \mathrm{a}$ and $8 \mathrm{~b}$. The variation of attenuation versus height over mixed paths of 3 sections along with the connected mixed path values of 2 sections and the homogeneous earth values.

(a) a land-sea-land path. (b) a sea-land-sea path.

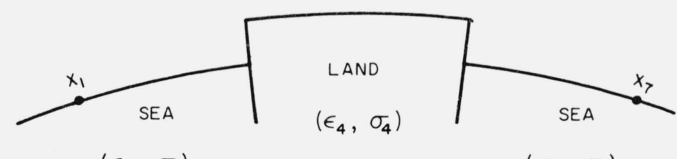

FIGURE 9. The geometry of an island having bluffs for $\left(\epsilon_{2}, \sigma_{2}\right)$

$\left(\epsilon_{6}, \sigma_{6}\right)$ figures $10 a$ and $10 b$.

Figure 10a shows the field strength in $\mathrm{dB}(0 \mathrm{~dB}=1 \mathrm{mV} / \mathrm{m})$ versus distance $(\mathrm{km})$ for several lengths of the island, when the $2\left|E_{0}\right|$ [defined by (1.1)] is assumed to be given by $300 /\left(r_{2}+r_{4}+r_{6}\right)$ $\mathrm{mV} / \mathrm{m}^{2} \quad$ Figure $10 \mathrm{~b}$ shows the corresponding phase delay in cycles. Here it may be noticed that the phase of $\boldsymbol{E}_{\mathbf{0}}$ defined by (1.1) is the phase delay corresponding to the distance measured along the surface of a mean earth radius $a$ (fig. 1). In figure $10 \mathrm{~b}$ and also in the following figures $11 \mathrm{~b}$ and $13 \mathrm{~b}$, the relative phase is displayed when this mean surface is taken at the sea level.

Figures 10a and 10b show the recovery effect as well as the diffraction loss in the vicinity of the bluff at the second coastline. The effect of the bluffs may also be seen by comparing to figures $11 \mathrm{a}$ and $11 \mathrm{~b}$, in which no bluff is assumed with the same values for other parameters as in figures $10 \mathrm{a}$ and $10 \mathrm{~b}$.

${ }^{2}$ This value is the field strength when the transmitter of a vertical electric dipole and the receiver are both placed on a perfectly conducting plane earth and the radiation power is $1 \mathrm{~kW}$. 

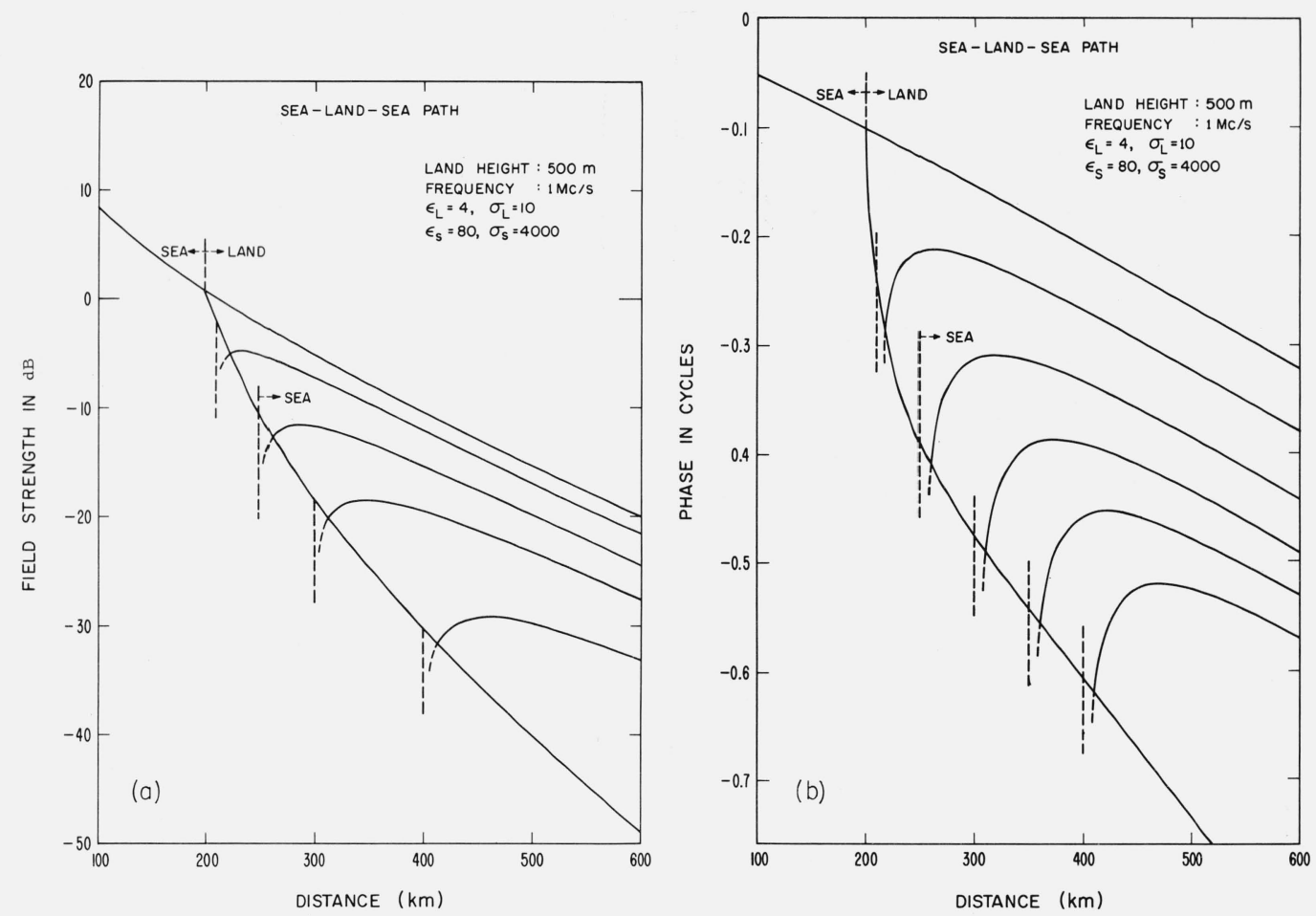

Figures 10a and 10b. An illustration of (a) the field strength and (b) the phase delay versus distance for island paths.

(The broken lines are the expected continuations of the solid lines for the $3 \mathrm{~d}$ section.)
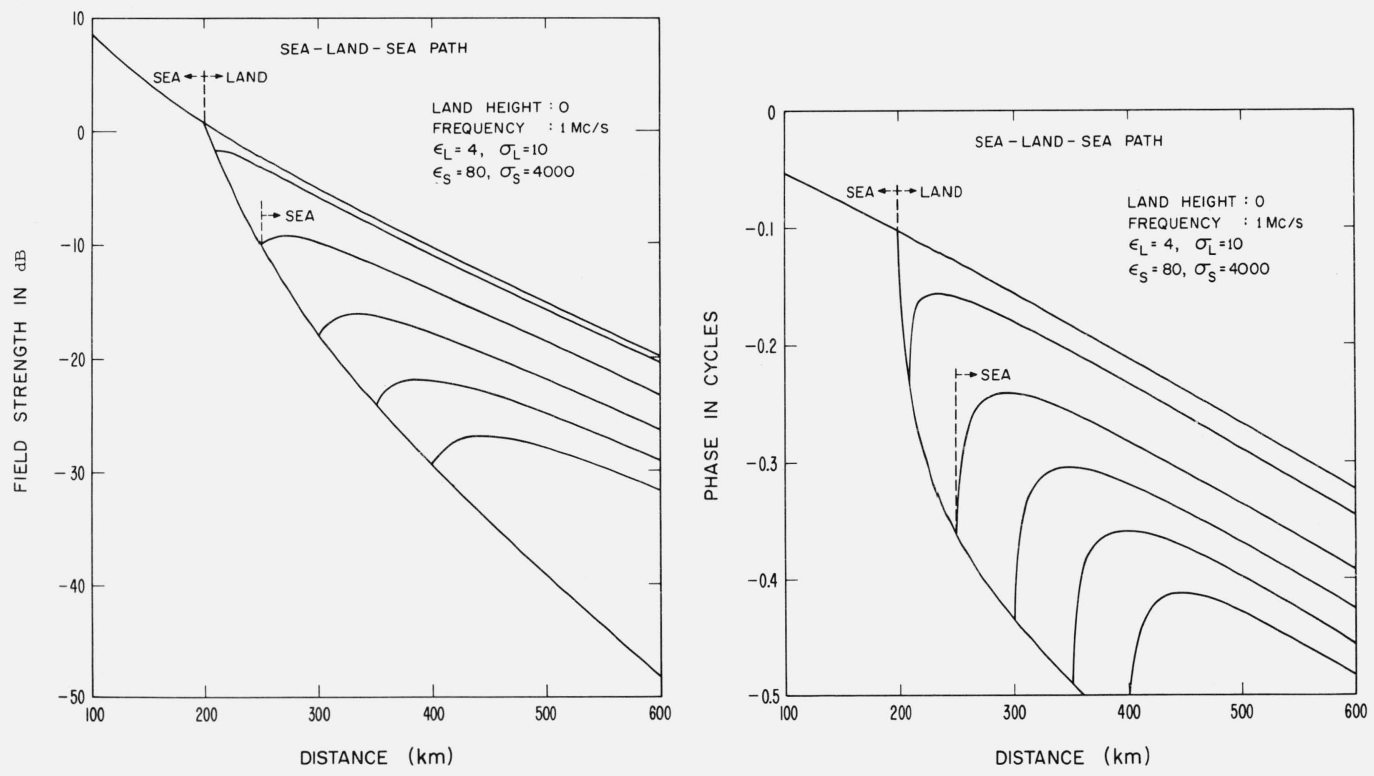

FigurEs 1la and 11b. (a) The field strength and (b) the phase delay versus distance when the island in figure 10 has no bluffs. 


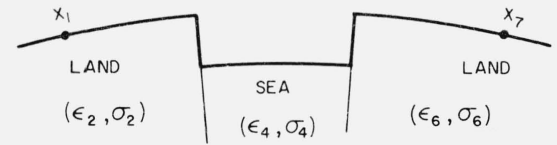

FiguRE 12. The geometry of a channel for figures $13 a$ and $13 b$.
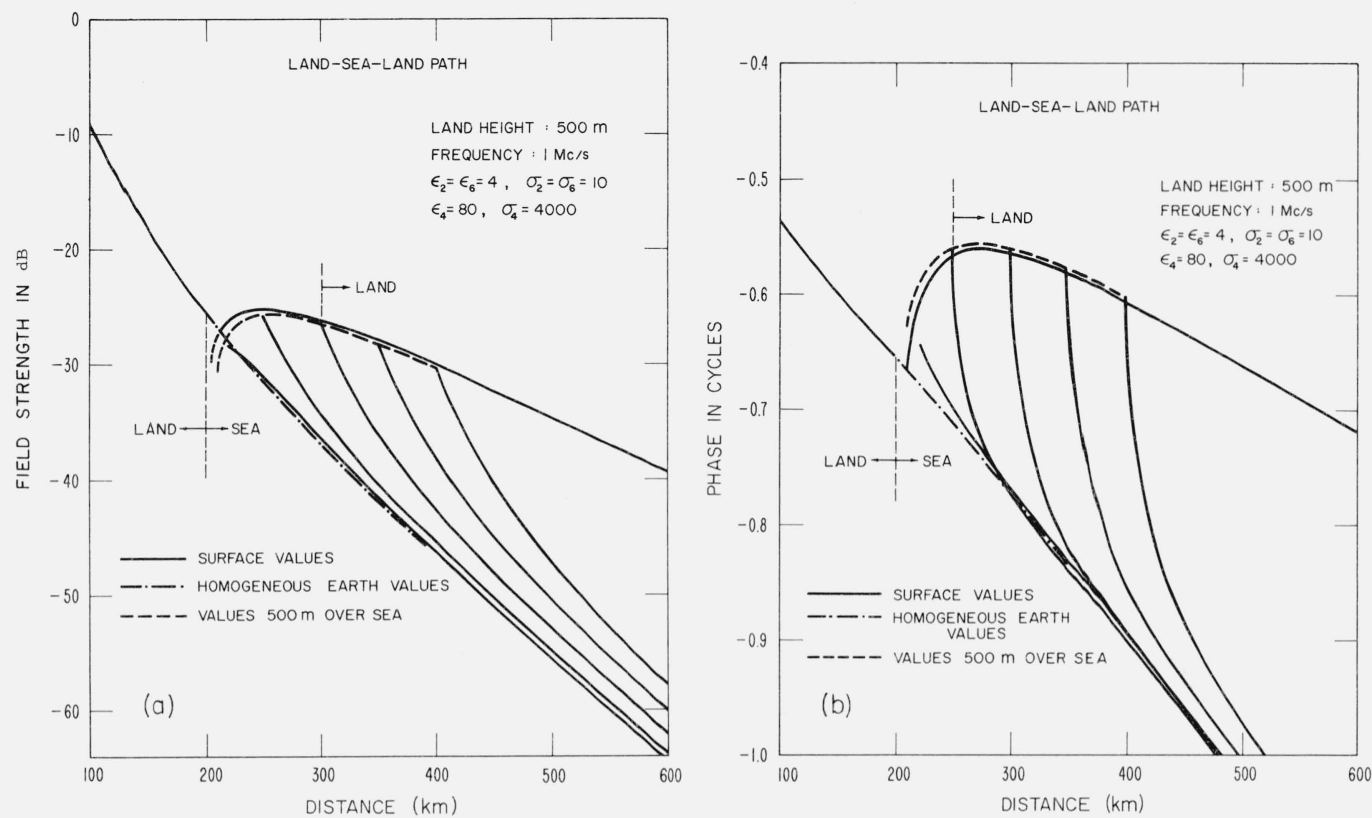

FiguRES 13a and 13b. An illustration of (a) the field strength and $(b)$ the phase delay versus distance for channel paths.

In figure 12, a land-sea-land path is illustrated, where the height of the land sides are again $500 \mathrm{~m}$ and the other conditions are assumed to be the same as in the preceding case of an island path. The solid lines in figure 13a show the field strength along the ground surface, while the broken line shows the field strength $500 \mathrm{~m}$ above the sea section, this being the same height as the land. Generally, the latter values would be lower than the former (up to about $6 \mathrm{~dB}$ ) except for the vicinities of the bluff where the diffraction loss becomes predominant, since a smaller contribution is expected from the reflected wave from the sea surface. Figure 13b shows the corresponding phase delay.

Finally, it may be noticed that, in figures 10, 11, and 13, the possible contribution of the skywave by the ionosphere is not taken into account, which becomes predominant at large distances [Wait and Walters, 1963].

\section{Conclusion}

Numerical results based on previous papers [Furutsu, 1957a, 1957b, 1963] are presented for propagation over an inhomogeneous earth consisting of three sections with different heights and electrical properties; a ridge may also be located at each section boundary. This is illustrated in figure 1.

The flat earth formula [Furutsu, 1955] is used to show the asymptotic approach of the spherical earth values and the corresponding flat earth values at short distances, although the flat earth formula is available only when the earth's surface is smooth and both the transmitter and the receiver 
are on the ground. A set of curves is illustrated for the flat earth values of the attenuation and the phase delay of land-sea-land paths. It shows the fact that, when the finite conductivity of the sea is taken into account and the Sommerfeld numerical distance of the sea part becomes of the order of magnitude of 1 or greater, the phase delay of the attenuation coefficient can exceed $\pi$ although it can not in the case of a homogeneous flat earth.

The effect of a mesa, as illustrated in figure 5, is considered and the values of the attenuation are compared with the corresponding values obtained when the obstacle is a ridge of the same height. The mesa and the knife edge both exhibit the effect of the obstacle gain, and the effect of the mesa length is found to become more predominant with increasing height.

The variation of attenuation is also illustrated as the receiver is raised when the path is a land-sea-land path or a sea-land-sea path; this situation is illustrated in figure 7 . As may be seen from this figure, the field strength computed over a three-section mixed path is expected to depend mostly on the sections closer to the transmitter as the receiver exceeds the heights of the optical boundary points. Thus the attenuation is compared with the mixed path values of two sections and further with the homogeneous earth values. However, the mutual approach of these values is found at much higher points than the corresponding optical boundary points.

Finally, in figures $10 \mathrm{a}, 10 \mathrm{~b}, 13 \mathrm{a}$, and $13 \mathrm{~b}$, the field strength and the phase delay are illustrated for a sea-land-sea path and a land-sea-land path, respectively, when the land is $500 \mathrm{~m}$ above the sea; both the transmitter and the receiver are on the ground and the frequency is $1 \mathrm{Mc} / \mathrm{s}$. The recovery effect as well as the diffraction loss in the vicinities of the bluffs at the coastlines are observed in these figures.

Although the present theory is valid only for terrains with sharp vertical faces, as illustrated in figure 1, it may be available for other cases, in the same way as the knife edge model has been found to be useful for actual ridge diffraction of VHF waves.

The author thanks R. E. Wilkerson, Perry H. Elder, and Raymond F. Hartmann for their help in obtaining the numerical results for this paper.

\section{Appendix $\AA$}

Putting

$$
\begin{gathered}
A=1-\sqrt{\pi k_{1}\left(r_{2}+r_{4}+r_{6}\right) / 2} e^{i \pi / 4} B, \\
\rho_{2}=-i\left(k_{1} / k_{2}^{\prime}\right)^{2} k_{1} r_{2} / 2, \quad \rho_{4}=-i\left(k_{1} / k_{4}^{\prime}\right)^{2} k_{1} r_{4} / 2, \quad \rho_{6}=-i\left(k_{1} / k_{6}^{\prime}\right)^{2} k_{1} r_{6} / 2
\end{gathered}
$$

[these notations $\rho_{m}$ 's are to be distinguished from the same notations used in (1.12)], then

$$
\begin{aligned}
& B=\frac{k_{1} k_{2}^{\prime} k_{6}^{\prime}}{k_{4}^{\prime}\left(k_{4}^{\prime}+k_{2}^{\prime}\right)\left(k_{4}^{\prime}+k_{6}^{\prime}\right)}\left\{\frac{2}{\pi} \tan ^{-1} \sqrt{\frac{m_{4}}{m_{2} m_{6}}} \mathscr{E}\left(i \sqrt{\rho_{4} / m_{4}}\right)\right. \\
& -\mathscr{E}\left(i \sqrt{\rho_{4} m_{2} / m_{4}}, \sqrt{\left.\left(m_{4}+m_{6}\right) / m_{2}\right)}-\mathscr{E}\left(i \sqrt{\rho_{4} m_{6} / m_{4}}, \sqrt{\left(m_{4}+m_{2}\right) / m_{6}}\right)\right. \\
& \left.+\mathscr{\delta}\left(i \sqrt{\rho_{4} m_{2} / m_{4}}, \sqrt{m_{6} / m_{2}}, \sqrt{m_{4} / m_{6}}\right)+\mathscr{E}\left(i \sqrt{\rho_{4} m_{6} / m_{4}}, \sqrt{m_{2} / m_{6}}, \sqrt{m_{4} / m_{2}}\right)\right\} \\
& +\frac{k_{1}}{\left(k_{4}^{\prime}+k_{2}^{\prime}\right)\left(k_{4}^{\prime}+k_{6}^{\prime}\right)}\left\{k_{6}^{\prime}\left(\mathscr{E}\left(i \sqrt{\rho_{4}\left(m_{4}+m_{6}\right) / m_{4}}\right)-\mathscr{E}\left(i \sqrt{\rho_{4} m_{6} / m_{4}}, \sqrt{m_{4} / m_{6}}\right)\right) \mathscr{E}\left(i \sqrt{\rho_{2}}\right)\right. \\
& +k_{2}^{\prime}\left(\mathscr{E}\left(i \sqrt{\rho_{4}\left(m_{4}+m_{2}\right) / m_{4}}\right)-\mathscr{E}\left(i \sqrt{\rho_{4} m_{2} / m_{4}}, \sqrt{m_{4} / m_{2}}\right)\right) \mathscr{E}\left(i \sqrt{\rho_{6}}\right)
\end{aligned}
$$




$$
\begin{aligned}
& \left.+k_{4}^{\prime} \mathscr{O}\left(i \sqrt{\rho_{2}}\right) \mathscr{E}\left(i \sqrt{\rho_{4}}\right) \mathscr{E}\left(i \sqrt{\rho_{6}}\right)\right\}+\left(\frac{k_{1}}{k_{2}^{\prime}+k_{6}^{\prime}}\right)\left\{\frac{k_{2}^{\prime}}{k_{6}^{\prime}} \mathscr{E}\left(i \sqrt{\rho_{6} / m_{6}}\right)\right.
\end{aligned}
$$

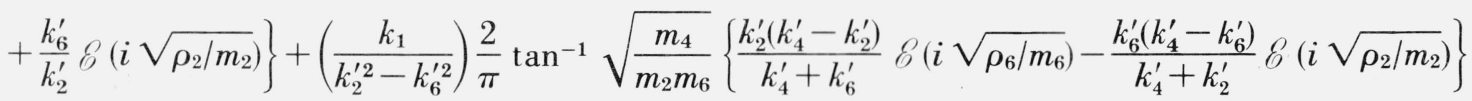

$$
\begin{aligned}
& +\left(\frac{k_{1}}{k_{2}^{\prime 2}-k_{6}^{\prime 2}}\right)\left[( \frac { k _ { 4 } ^ { \prime } - k _ { 6 } ^ { \prime } } { k _ { 4 } ^ { \prime } + k _ { 2 } ^ { \prime } } ) \left\{k_{2}^{\prime} \mathscr{E}\left(i \sqrt{\rho_{2}\left(m_{4}+m_{2}\right) / m_{2}}\right) \mathscr{E}\left(i \sqrt{\rho_{6}}\right)\right.\right. \\
& \left.\left.-k_{6}^{\prime} \delta\left(i \sqrt{\rho_{2} m_{6} / m_{2}}, \sqrt{\left(m_{4}+m_{2}\right) / m_{6}}\right)\right\}-\frac{k_{2}^{\prime}\left(k_{2}^{\prime} k_{4}^{\prime}-k_{6}^{\prime 2}\right)}{k_{6}^{\prime}\left(k_{4}^{\prime}+k_{6}^{\prime}\right)} \delta\left(i \sqrt{\rho_{6}\left(m_{4}+m_{2}\right) / m_{6}}, \sqrt{m_{6} /\left(m_{4}+m_{2}\right)}\right)\right] \\
& +\left(\frac{k_{1}}{k_{6}^{\prime 2}-k_{2}^{\prime 2}}\right)\left[( \frac { k _ { 4 } ^ { \prime } - k _ { 2 } ^ { \prime } } { k _ { 4 } ^ { \prime } + k _ { 6 } ^ { \prime } } ) \left\{k_{6}^{\prime} \mathscr{E}\left(i \sqrt{\rho_{6}\left(m_{4}+m_{6}\right) / m_{6}}\right) \mathscr{E}\left(i \sqrt{\rho_{2}}\right)\right.\right. \\
& \left.-k_{2}^{\prime} \mathscr{E}\left(i \sqrt{\rho_{6} m_{2} / m_{6}}, \sqrt{\left.\left(m_{4}+m_{6}\right) / m_{2}\right)}\right\}-\frac{k_{6}^{\prime}\left(k_{6}^{\prime} k_{4}^{\prime}-k_{2}^{\prime 2}\right)}{k_{2}^{\prime}\left(k_{4}^{\prime}+k_{2}^{\prime}\right)} \delta\left(i \sqrt{\rho_{2}\left(m_{4}+m_{6}\right) / m_{2}}, \sqrt{m_{2} /\left(m_{4}+m_{6}\right)}\right)\right] \\
& +\left(\frac{k_{1}}{k_{2}^{\prime 2}-k_{6}^{\prime 2}}\right)\left[( \frac { k _ { 4 } ^ { \prime } - k _ { 2 } ^ { \prime } } { k _ { 4 } ^ { \prime } + k _ { 6 } ^ { \prime } } ) \left\{k_{6}^{\prime} \mathscr{E}\left(i \sqrt{\rho_{2}}\right) \mathscr{E}\left(i \sqrt{\rho_{6} m_{4} / m_{6}}, \sqrt{m_{6} / m_{4}}\right)\right.\right. \\
& \left.-k_{2}^{\prime} \mathscr{\delta}\left(i \sqrt{\rho_{6} m_{2} / m_{6}}: \sqrt{m_{4} / m_{2}}, \sqrt{m_{6} / m_{4}}\right)\right\}
\end{aligned}
$$

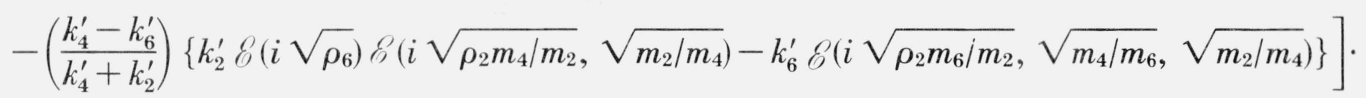

\section{Appendix B}

The function $\overline{\mathscr{E}}(z, \beta, \alpha)$ can be given in terms of the $\chi$-function using the relation (1.11c). Therefore, in this appendix, the analytical expansions will be given only for the functions $\delta(z)$, $\delta(z, \alpha)$ and $\chi(z, \alpha, \beta)$.

When $\alpha$ and $\beta$ are real positive, the definitions of these functions in (1.11a) give the following expressions:

$$
\begin{aligned}
& \mathscr{E}(z)=e^{z^{2}}-\bar{\delta}(z), \\
& \mathscr{\delta}(z, \alpha)=\frac{2}{\pi}\left(\tan ^{-1} \alpha^{-1}\right) e^{\left(1+\alpha^{2}\right) z^{2}}-e^{\alpha^{2} z^{2}} \overline{\mathscr{\delta}}(z)+\bar{\delta}(z, \alpha) . \\
& \chi(z, \beta, \alpha)=\chi(z, \alpha, \beta)=e^{\beta^{2} z^{2}} \overline{\mathscr{\delta}}(z, \alpha)+e^{\alpha^{2} z^{2}} \overline{\mathscr{\delta}}(z, \beta) \\
& +\chi(0, \alpha, \beta) e^{\left(1+\alpha^{2}+\beta^{2}\right) z^{2}}-e^{\left(\alpha^{2}+\beta^{2}\right) z^{2}} \overline{\mathscr{E}}(z)-\chi(z, \alpha, \beta) .
\end{aligned}
$$


The power series expansions of $\overline{\mathscr{C}}(z), \overline{\mathscr{C}}(z, \alpha)$, and $\bar{\chi}(z, \alpha, \beta)$ with respect to $z$ are given by

$$
\begin{aligned}
& \overline{\mathscr{E}}(z)=\sum_{n=1}^{\infty} c_{n}, \\
& c_{n}=(n-1 / 2)^{-1} z^{2} c_{n-1}, \quad c_{1}=(2 / \sqrt{\pi}) z, \\
& \overline{\mathscr{O}}(z, \alpha)=\sum_{n=1}^{\infty} d_{n}, \\
& d_{n}=n^{-1}\left\{\left(1+\alpha^{2}\right) z^{2} d_{n-1}+f_{n}\right\}, \\
& f_{n}=(n-1 / 2)^{-1} \alpha^{2} z^{2} f_{n-1}, \quad d_{1}=f_{1}=(2 / \pi) \alpha z^{2}, \\
& \bar{\chi}(z, \alpha, \beta)=\sum_{n=1}^{\infty} x_{n}, \\
& x_{n}=(n+1 / 2)^{-1}\left\{\left(1+\alpha^{2}+\beta^{2}\right) z^{2} x_{n-1}+y_{n}\right\}, \\
& y_{n}=n^{-1}\left\{\left(\alpha^{2}+\beta^{2}\right) z^{2} y_{n-1}+a_{n}+b_{n}\right\}, \\
& a_{n}=(n-1 / 2)^{-1} \alpha^{2} z^{2} a_{n-1}, \quad b_{n}=(n-1 / 2)^{-1} \beta^{2} z^{2} b_{n-1}, \\
& a_{1}=b_{1}=\left(2 / \sqrt{\pi^{3}}\right) \alpha \beta z^{3} .
\end{aligned}
$$

While (Bl) gives the power series expansions with respect to $z$, the asymptotic expansions for large values of $z$ are found to be given by the following equations:

$$
\begin{aligned}
& \mathscr{E}(z) \sim \sum_{n=0}^{\infty} g_{n}, \quad|z|>>1, \quad|\arg (z)|<3 \pi / 4, \quad g_{n}=-\frac{n-1 / 2}{z^{2}} g_{n-1}, \quad g_{0}=\frac{1}{\sqrt{\pi} z}, \quad \text { (B6) } \\
& \mathscr{E}(z, \alpha) \sim \frac{1}{\sqrt{\pi}\left(1+\alpha^{2}\right) z} \sum_{n=0}^{\infty} j_{n}\left(\alpha / \sqrt{1+\alpha^{2}}, R\right), \quad R \equiv \sqrt{1+\alpha^{2}} z, \quad|R|>>1, \quad|\arg (R)|<3 \pi / 4,
\end{aligned}
$$
$\chi(z, \alpha, \beta) \sim \frac{1}{\sqrt{\pi}\left(1+\alpha^{2}+\beta^{2}\right) z} \sum_{n=0}^{\infty} h_{n}$

$$
R \equiv \sqrt{1+\alpha^{2}+\beta^{2}} z, \quad|R|>>1, \quad|\arg (R)|<3 \pi / 4,
$$

with

$$
\begin{aligned}
& h_{0}=\mathscr{E}(\alpha z) \mathscr{E}(\beta z), \quad h_{n+1}=\frac{\alpha^{2}+\beta^{2}}{1+\alpha^{2}+\beta^{2}} h_{n}-\frac{n}{1+\alpha^{2}+\beta^{2}} \frac{1}{2 R^{2}} h_{n-1} \\
& -\frac{1}{\sqrt{\pi} R}\left\{\frac{\alpha}{\sqrt{1+\alpha^{2}+\beta^{2}}} j_{n}\left(\beta / \sqrt{1+\alpha^{2}+\beta^{2}}, R\right)+\frac{\beta}{\sqrt{1+\alpha^{2}+\beta^{2}}} j_{n}\left(\alpha / \sqrt{1+\alpha^{2}+\beta^{2}}, R\right)\right\}
\end{aligned}
$$

Here the $j_{n}(\gamma, R)$ 's used in (B1) and (B8) are defined by

$$
\begin{aligned}
j_{0} & =\mathscr{E}(\gamma R), \\
j_{2 n+1} & =B j_{2 n}-n A j_{2 n-1}-k_{n},
\end{aligned}
$$




$$
\begin{aligned}
& j_{2 n}=B j_{2 n-1}-\left(n-\frac{1}{2}\right) A j_{2 n-2}, \\
& k_{n}=-\left(n-\frac{1}{2}\right) R^{-2} k_{n-1}, \quad k_{0}=\pi^{-1 / 2} \gamma R^{-1}, \\
& A=\left(1-\gamma^{2}\right) R^{-2}, \quad B=\gamma^{2} .
\end{aligned}
$$

In all the above equations, both $\alpha$ and $\beta$ are assumed to be real and positive. However, these equations may also be used for complex values of $\alpha$ and $\beta$ if the proper formulas are used. These formulas may be found using analytic continuation.

\section{References}

Bremmer, H. (1954), The extension of Sommerfeld's formula for the propagation of radio waves over a flat earth to different conductivities of the soil, Physica XX, 441 .

Feinberg, E. L. (1944), On the propagation of radio waves along an imperfect surface, J. Phys. IX, No. 1.

Feinberg, E. L. (1946), On the propagation of radio waves along an imperfect surface, J. Phys. X, No. 5.

Feinberg, E. L. (1959), Propagation of radio waves along an inhomogeneous surface, Nuovo Cimento 11 , 60-91.

Furutsu, K. (1955), Propagation of electromagnetic waves over flat earth across two boundaries separating three different media, J. Radio Res. Lab. (Japan) 2, 239-279.

Furutsu, K. (1957a), Wave propagation over an irregular terrain, Part I, J. Radio Res. Lab. 4, 135-153.

Furutsu, K. (1957b), Wave propagation over an irregular terrain, Part II, J. Radio Res. Lab. 4, 349-393.

Furutsu, K. (1959), Wave propagation over an irregular terrain, Part III, J. Radio Res. Lab. 6, 71-102.

Furutsu, K. (1963), On the theory of radio wave propagation over inhomogeneous earth, J. Res. NBS 67D (Radio Prop.), No. $1,39-62$.

Furutsu, K., R. E. Wilkerson and R. F. Hartmann (1964), Some numerical results based on theory of radio wave propagation over inhomogeneous earth, Radio Sci. J. Res. NBS 68D, No. 7, 827-846.

Wait, J. R. (1961), On the theory of mixed-path ground wave propagation on a spherical earth, J. Res. NBS 65D (Radio Prop.), No. 4, 401-410.

Wait, J. R., and J. E. Householder (1956), Mixed-path ground wave propagation: 1. Short distance, J. Res. NBS 57, 1-15, RP2687.

Wait, J. R., and J. E. Householder (1957), Mixed-path ground wave propagation: 2. Larger distance, J. Res. NBS 59, 19-26, RP2770.

Wait, J. R., and L. C. Walters (1963), Curves for ground wave propagation over mixed land and sea paths, IEEE Trans. AP-1 1, No. 1, 38-45.

(Paper 69D7-536) 